\title{
Improving reliability of distribution networks using plug-in electric vehicles and demand response
}

\author{
Omid SADEGHIAN ${ }^{1}$, Morteza NAZARI-HERIS ${ }^{1}{ }^{10}$, Mehdi ABAPOUR ${ }^{1}$, \\ S. Saeid TAHERI ${ }^{2}$, Kazem ZARE ${ }^{1}$
}

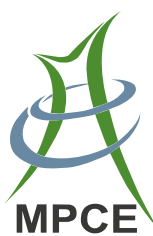

\begin{abstract}
Nowadays, utilities aim to find methods for improving the reliability of distribution systems and satisfying the customers by providing the continuity of power supply. Different methodologies exist for utilities to improve the reliability of network. In this paper, demand response (DR) programs and smart charging/discharging of plug-in electric vehicles (PEVs) are investigated for improving the reliability of radial distribution systems adopting particle swarm optimization (PSO) algorithm. Such analysis is accomplished due to the positive effects of both DR and PEVs for dealing with emerging challenges of the world such as fossil fuel reserves reduction, urban air pollution and greenhouse gas emissions. Additionally, the prioritization of DR and PEVs is presented for improving the reliability and analyzing the characteristics of
\end{abstract}

CrossCheck date: 25 January 2019

Received: 29 May 2018/Accepted: 25 January 2019/Published online: 7 May 2019

(C) The Author(s) 2019

$\triangle$ Morteza NAZARI-HERIS

mnazari.heris@gmail.com

Omid SADEGHIAN

Omidsadeghian1991@gmail.com

Mehdi ABAPOUR

abapour@tabrizu.ac.ir

S. Saeid TAHERI

ss.taheri@gmail.com

Kazem ZARE

kazem.zare@tabrizu.ac.ir

1 Faculty of Electrical and Computer Engineering, University of Tabriz, Tabriz, Iran

2 Department of Electrical Engineering, Faculty of Engineering, Sarab Branch, Islamic Azad University, Sarab, Iran distribution networks. The reliability analysis is performed in terms of loss of load expectation (LOLE) and expected energy not served (EENS) indexes, where the characteristics contain load profile, load peak, voltage profile and energy loss. Numerical simulations are accomplished to assess the effectiveness and practicality of the proposed scheme.

Keywords Plug-in electric vehicles (PEVs), Demand response (DR), Reliability improvement, Electric distribution system, Expected energy not served (EENS)

\section{Introduction}

The reliability of power delivered to customers has become more and more important in recent years. It is viewed as one of indexes for power quality of distribution network especially considering its impact on customer satisfaction. Utilities have many choices for improving the reliability of distribution network such as network reconfiguration, installation of energy storage systems and application of distributed generation resource (i.e., renewable and nonrenewable), utilization of plug-in electric vehicles (PEVs) by smart charging/discharging and implementation of demand response (DR) programs. The two latter choices have much potential for improving the reliability indexes of the distribution system.

Despite the superiority of electric vehicles to traditional ones in several indexes such as high efficiency, less air pollution and less noise creation, the network will be placed in front of peak load enhancement by increasing the penetration percentage of PEVs. Coordination of PEVs and optimal charging/discharging of such vehicles as well as supplying daily required energy of PEVs for daily trip can 
handle the challenge of peak load and even provide power for improving the reliability of network especially in smart grids, which requires higher reliability [1]. Battery utilization of PEVs for improving reliability prevents system operators to construct new power plants in a long term, which is effective in economic aspects.

On the other hand, DR has positive effect on reliability of distribution system. According to the definition given in [2], DR is the ability of consumers for improving paradigm of energy consumption to attain suitable prices and reliability improvement of the network. Vast advantages will be achieved for customers, utilities and also environment by implementing DR programs. Undeniable impacts of DR on the cost and reliability of power system tend to increase the adaptation of such program by utilities among their activities in recent years. Due to the sensitivity of demand to price, a slight decline in demand has great effect on the electricity price [3].

Different modellings for DR are accomplished in different studies using various approaches and objectives $[4,5]$. Utilizing PEVs in DR program is investigated in some studies with aims such as voltage control [6] and power balance [7]. Impact analysis of DR is accomplished in some studies for network characteristics such as load and voltage [8] and loss and electricity price [9]. Reliability improvement of power network with DR is done in recent studies [10-12]. Implementing DR in a real case study to prove the significant impact of such program in improving the reliability and retaining stability of network compared with conventional methods is accomplished in [10]. An optimization approach based on the reliability of generation units' failure is presented in [11], which is modeled based on the forced outage rate by investigating its effect on the indexes value of lost load and expected load not served. An approach for evaluating the role of price-based DR on the supply adequacy of smart distribution system is presented in [12] by introducing a method to increase the willingness of customers to participate in DR. Unlike the existing methods, the varying availability in the capability of customer for DR is considered in this paper.

Utilizing PEVs in different fields is investigated in literature. Several review studies are in the area of PEVs concentrating on smart charging procedures [13], technical challenges [14], impact of different PEV types and charging strategies on distribution network [15], computational scheduling methods for integrating this vehicles with grid [16] and recent trends in optimization techniques for hybrid vehicles and charging infrastructures [17]. Impact of PEVs on the system is investigated in some studies aiming to loss minimization [18], load variance smoothening [19] and stability [20, 21]. Reliability studies of distribution system in consequence of adding PEVs are presented in [22-24]. In [22], a new model is presented for direct optimization of reliability indexes by optimizing the charging/discharging of PEVs in power network and investigating a new techno-economic assessment. Reliability evaluation of distribution system in presence of PEVs is proposed in [23] considering two topologies (centralized and dispersed) for load points to reduce the energy not served in grid-connected mode and support the grid in islanding mode with the aim of maximizing energy exchange among load points by sectionalizing the failure parts of the grid. In [24], the impact of PEVs charging stations on reliability of distribution system for smoothening the system load is investigated, introducing expected energy not charged.

In the previous studies, the impact of both DR and PEVs have not been investigated simultaneously as two largescale factors for reliability improvement. To fill such gap, the performance of radial distribution system is improved in this paper by using PEVs and DR considering constraints of PEV batteries, daily energy consumption of such vehicles and charger size. Also, the penetration percentage of PEVs is improved, and PEVs and DR are prioritized in distribution networks. Additionally, network characteristics including load profile, load peak, voltage profile and loss are investigated in the aspect of reliability improvement. DR program is a real-time-pricing (RTP) program considering different participation percentage, which is studied in order to compare the obtained results. Also, both DR and optimized DR (ODR) are compared in reliability viewpoint. The well-known indexes of loss of load expectation (LOLE) and expected energy not served (EENS) are adopted for reliability studies. The particle swarm optimization (PSO) algorithm is adopted for optimizing objective functions of mentioned reliability indexes due to the nonlinearity of problem and the numerous advantages of this technique over mathematical methods. Moreover, a new direct formulation for calculating the capacity outage probability table (COPT) is proposed for the first time. Additionally, an approach for connecting PEVs to the buses of radial network is presented. The simulation results are provided and investigated in order to evaluate the performance of the proposed model by adopting IEEE 33-bus radial distribution networks.

The remainder of this study is organized as follows: the formulation of the proposed scheme is presented in Section 2. Section 3 contains the case studies and simulation results of the proposed model. Reliability improvement prioritization of the mentioned reliability indexes is provided in Section 4. Finally, the paper is concluded in Section 5 . 


\section{Problem formulation}

\subsection{DR modelling}

One of reliability improvement methods in distribution system is implementing DR by utilities, which was firstly proposed in [25]. In addition, the development of such concept and modeling of DR is accomplished in [3]. The sensitivity of the demand to price is called elasticity $(E)$ that represents the changing in demand for a unit of changing in the price of electricity as formulated in (1). Moreover, (2) defines the mutual-elasticity for $i^{\text {th }}$ hour to $j^{\text {th }}$ hour [26].

$E=\frac{\rho_{0}}{d_{0}} \frac{\partial d}{\partial \rho}$

$E_{h, j}=\frac{\rho_{j}^{0}}{L_{h}^{0}} \frac{\partial L_{h}}{\partial \rho_{j}}$

where $\rho_{j}^{0}$ and $\rho_{j}$ show the electricity price before and after implementing DR at $j^{\text {th }}$ hour, respectively, and $L_{h}^{0}, L_{h}$ are system load at $h^{\text {th }}$ hour before and after implementing DR.

Demands can be categorized according to elasticity. The first type of category (self-elasticity) shows the loads which can only be on or off and unable to move from one hour to other hours such as illumination loads that have negative elasticity. On the other hand, the second type (cross-elasticity) represents the loads that can be displaced from peak hours to flat or valley hours which have positive value [27].

The elasticity is as (3), where elements of the main diagonal are represented for self-elasticity while other elements of this parameter show the cross-elasticity [28].

$\boldsymbol{E}=\left[\begin{array}{cccccc}E_{1,1} & E_{1,2} & \ldots & E_{1, j} & \ldots & E_{1,24} \\ E_{2,1} & E_{2,2} & \ldots & E_{2, j} & \ldots & E_{2,24} \\ \vdots & \vdots & & \vdots & & \vdots \\ E_{h, 1} & E_{h, 2} & \ldots & E_{h, j} & \ldots & E_{h, 24} \\ \vdots & \vdots & & \vdots & & \vdots \\ E_{24,1} & E_{24,2} & \ldots & E_{24, j} & \ldots & E_{24,24}\end{array}\right]$

Different models are designed for DR including linear models [29] and nonlinear models [30]. It is obvious that nonlinear models can model the load better than linear models. In [30], numerous nonlinear models such as power, exponential and logarithmic models are presented and show that the power model has a lower risk compare to other nonlinear models (i.e., exponential and logarithmic) for implementing. Accordingly, this model is adopted in this paper.
The adopted DR model in this research is according to (4). Considering participation percentage $P_{P}$ of consumers, the previous equation will be revised as (5). So, in this situation, only a fraction of load participates in DR program, for which the participation percentage is in range of [0 100].

$L_{h}=L_{h}^{0}\left[\prod_{j=1}^{24}\left(\frac{\rho_{j}}{\rho_{j}^{0}}\right)^{E_{h, j}}\right]$

$L_{h}=\frac{P_{P}}{100} L_{h}^{0}\left[\prod_{j=1}^{24}\left(\frac{\rho_{j}}{\rho_{j}^{0}}\right)^{E_{h, j}}\right]+\left(1-\frac{P_{P}}{100}\right) L_{h}^{0}$

It is assumed that the price is allowed to be changed $20 \%$ more in peak hours $P_{h}$ and 20\% less in valley hours $V_{h}$ than the original price as follows:

$\rho_{h}=\left\{\begin{aligned} 0.8 \rho_{h}^{0} & \rho_{h}^{0} \in V_{h} \\ 1.2 \rho_{h}^{0} & \rho_{h}^{0} \in P_{h}\end{aligned}\right.$

In the case of applying ODR instead of normal DR, the following constraint is considered:

$\sum_{h=1}^{H} \rho_{h}=\sum_{h=1}^{H} \rho_{h}^{0}$

\subsection{Smart charging/discharging of PEVs}

As mentioned earlier, reliability studies of distribution system contains well-known indexes of LOLE and EENS which are important for costumers. LOLE determines the probability of total expected time, where power is not supplied to customers. On the other hand, EENS represents the total value of expected energy not supplied in the outage time. This outage time is determined in LOLE. Calculation of these two indexes in presence of PEVs will be changed to optimization problems that are formulated from the concepts of these indexes [31], which is presented in [22]. So, the objective functions for LOLE and EENS can be stated as (8) and (9), respectively. The formulations of $t_{s, h}$ and $w_{s, h}$ is according to (10) and (11), respectively.

$$
\begin{aligned}
& \text { LOLE }=\min \sum_{s=1}^{S}\left(p_{s} \sum_{h=1}^{H} t_{s, h}\right) \\
& \text { EENS }=\min \sum_{s=1}^{S}\left(p_{s} \sum_{h=1}^{H} w_{s, h}\right) \\
& t_{s, h}= \begin{cases}1 & P_{s}-\left[L_{h}+N\left(C_{h}-D_{h}\right)\right]<0 \\
0 & P_{s}-\left[L_{h}+N\left(C_{h}-D_{h}\right)\right] \geq 0\end{cases}
\end{aligned}
$$


$w_{s, h}= \begin{cases}{\left[L_{h}+N\left(C_{h}-D_{h}\right)\right]-P_{s}} & P_{s}-\left[L_{h}+N\left(C_{h}-D_{h}\right)\right]<0 \\ 0 & P_{s}-\left[L_{h}+N\left(C_{h}-D_{h}\right)\right] \geq 0\end{cases}$

where $H$ is the total hours, which is 24 in this paper; $N$ is the total number of PEVs; $C_{h}, D_{h}$ are the charging and discharging mean power of one PEV at $h^{\text {th }}$ hour; $S$ is the total number of state $2^{G}$; and $p_{s}, P_{s}$ are the probability and installed capacity of $s^{\text {th }}$ states of availability and unavailability of generation units, respectively. To determine these two parameters, firstly the COPT of generation units should be formed. To this end, all possible states for availability of generation units must be generated as shown in Table 1.

This table forms $I_{s, g}$, where $g$ is the indexes for generation units and $G$ is the total number of generation units. $I_{s, g}$ is 1 when $g^{\text {th }}$ unit in $s^{\text {th }}$ state is available and is 0 when such unit is not available. This parameter is determined by:

$I_{s, g}= \begin{cases}0 & 0<\bmod \left(\frac{s}{2^{g}}\right) \leq \frac{2^{g}}{2} \\ 1 & \bmod \left(\frac{s}{2^{g}}\right)=0 \text { or } \quad \bmod \left(\frac{s}{2^{g}}\right)>\frac{2^{g}}{2}\end{cases}$

Finally, for obtaining $p_{s}$ and $P_{s}$, the following equations are proposed:

$p_{s}=\prod_{g=1}^{G}\left[A_{g} I_{s, g}+\left(1-A_{g}\right)\left(1-I_{s, g}\right)\right] \quad \forall s$

$P_{s}=\sum_{g=1}^{G} P_{g}^{\max } I_{s, g} \quad \forall s$

where $P_{g}^{\max }$ and $A_{g}$ are installed capacity and availability of $g^{\text {th }}$ generation unit, respectively.

Table 1 All probable states for availability of generation units

\begin{tabular}{llllll}
\hline$s$ & $I_{s, g}$ & & & & \\
\cline { 2 - 6 } & $g=1$ & $g=2$ & $g=3$ & $\ldots$ & $g=G$ \\
\hline$s=1$ & 0 & 0 & 0 & $\ldots$ & 0 \\
$s=2$ & 1 & 0 & 0 & $\ldots$ & 0 \\
$s=3$ & 0 & 1 & 0 & $\ldots$ & 0 \\
$s=4$ & 1 & 1 & 0 & $\ldots$ & 0 \\
$s=5$ & 0 & 0 & 1 & $\ldots$ & 0 \\
$s=6$ & 1 & 0 & 1 & $\ldots$ & 0 \\
$s=7$ & 0 & 1 & 1 & $\ldots$ & 0 \\
$s=8$ & 1 & 1 & 1 & $\ldots$ & 0 \\
$\vdots$ & $\vdots$ & $\vdots$ & $\vdots$ & $\vdots$ & $\vdots$ \\
$s=S$ & 1 & 1 & 1 & 1 & 1 \\
\hline
\end{tabular}

Considering the constant size of charger $R$ in real, the time of connecting PEVs to grid for charging/discharging is as follows:

$T_{h}^{C}=\frac{C_{h}}{R} \times 60$

$T_{h}^{D}=\frac{D_{h}}{R} \times 60$

where $T_{h}^{C}$ and $T_{h}^{D}$ are the time of charging and discharging at $h^{\text {th }}$ hour, respectively. In addition, the energy of charging/discharging are as (17) and (18), which are considered as the constraints of battery:

$E_{h}^{C}=C_{h}=\frac{T_{h}^{C}}{60} R$

$E_{h}^{D}=D_{h}=\frac{T_{h}^{D}}{60} R$

This optimization problem is solved subjected to the following constraints:

$E_{h}^{B}=E_{h-1}^{B}+N\left(\eta_{C} E_{h}^{C}+\frac{E_{h}^{D}}{\eta_{D}}-\delta_{h}^{e}\right)$

$E_{h}^{B}>\delta_{h+1}^{e}$

$0<E_{h}^{B}<N C_{B}$

$0<C_{h}<R$

$0<D_{h}<R$

$\delta_{h}^{e}=\frac{\delta_{h}}{W}$

where $E_{h}^{B}$ is the energy of PEV batteries at the end of hour $h ; \eta_{C}$ and $\eta_{D}$ are the charging/discharging efficiencies of batteries; $\delta_{h}^{e}$ is the electricity energy consumed by one PEV in $h^{\text {th }}$ hour; $\delta_{h}$ is the distance driven by one PEV in $h^{\text {th }}$ hour; $W$ is the discharging conversion factor (i.e. a distance) which can be driven with $1 \mathrm{kWh}$ by one PEV; and $C_{B}$ is the capacity of each PEV's battery.

\subsection{The proposed approach for connecting PEVs to buses}

In this approach, each PEV is connected to one of the buses in radial distribution system based on a probability. This probability is based on the load of buses. Assuming that $P_{z}$ shows the load of $z^{\text {th }}$ bus in radial distribution system. Firstly, the cumulative load of each bus is determined by summing the loads from bus 1 to the corresponding bus using (25), where $C L_{b}$ shows the cumulative load of $b^{\text {th }}$ bus from bus 1 to bus $b$. 
$C L_{b}=\sum_{z=1}^{b} P_{z} \quad b=1,2, \ldots, B$

For each PEV, a random number $u_{n}$ is generated using uniform distribution which is in range of $\left(\begin{array}{ll}0 & 1\end{array}\right)$. So $C L_{1}<u_{n} \cdot C L_{B}<C L_{B}$ and thus support all the buses. Finally, (26) is utilized for connecting each PEV to resulted bus of it, where $C B_{n}^{h}$ represents the connecting bus of $n^{\text {th }} \mathrm{PEV}$ in $h^{\text {th }}$ hour.

$C B_{n}^{h}=b \mid\left\{u_{n} \cdot C L_{B} \in\left(C L_{b-1}, C L_{b}\right), b \in(2,3, \ldots, B)\right\}$

\subsection{Load flow and power loss calculation}

Load flow method for voltage and loss calculation in this approach is backward-forward technique, which is basedon the direct application of the Kirchhoff's voltage law (KVL) and Kirchhoff's circuit law (KCL) rules [32].

\subsection{PSO algorithm description}

The PSO algorithm is a population-based stochastic optimization technique, which has been founded based on the swarm-intelligence [33], and it is improved in [34]. Faster convergence, low memories requirement, less parameters to tune, usability in problems with large and

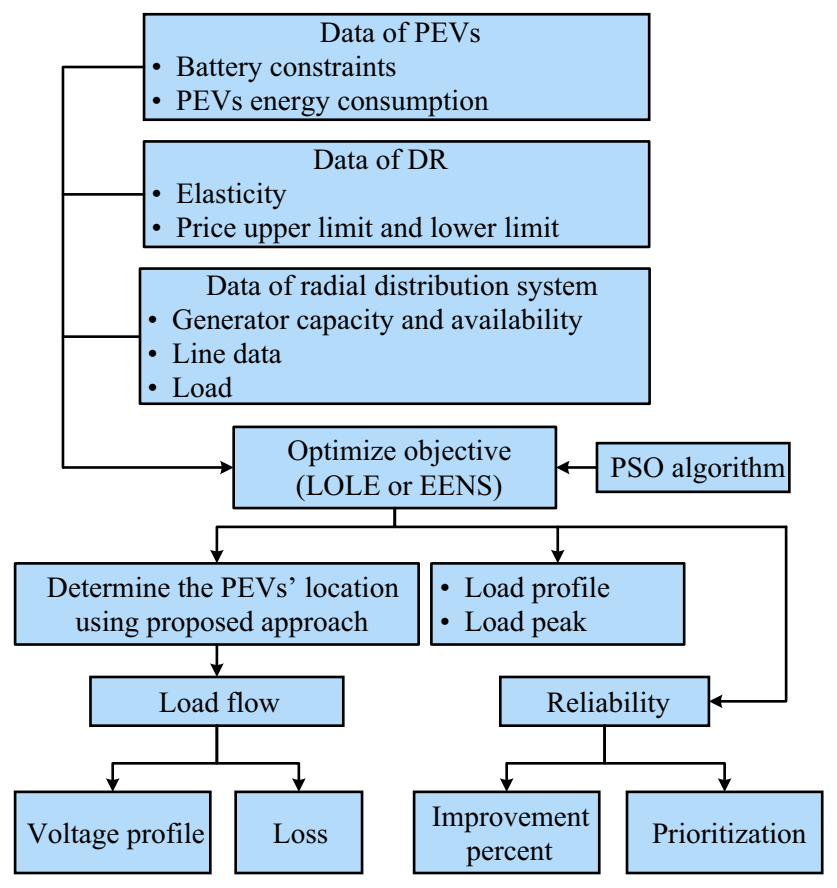

Fig. 1 Diagram of the proposed framework

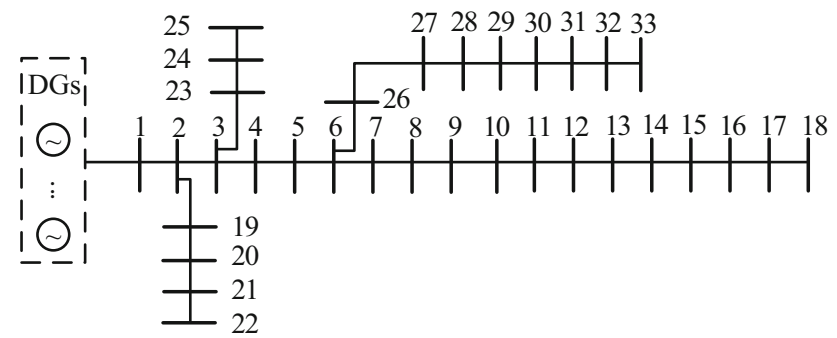

Fig. 2 IEEE 33-bus radial distribution test system

complex search spaces, capability of solving many nonlinear hard optimization problems, no need to any gradient information about the objective or error function, and low dependency on the initial solution in order to get the global optimal solution are the advantages of PSO. Diagram of the proposed scheme for reliability improvement is presented in Fig. 1.

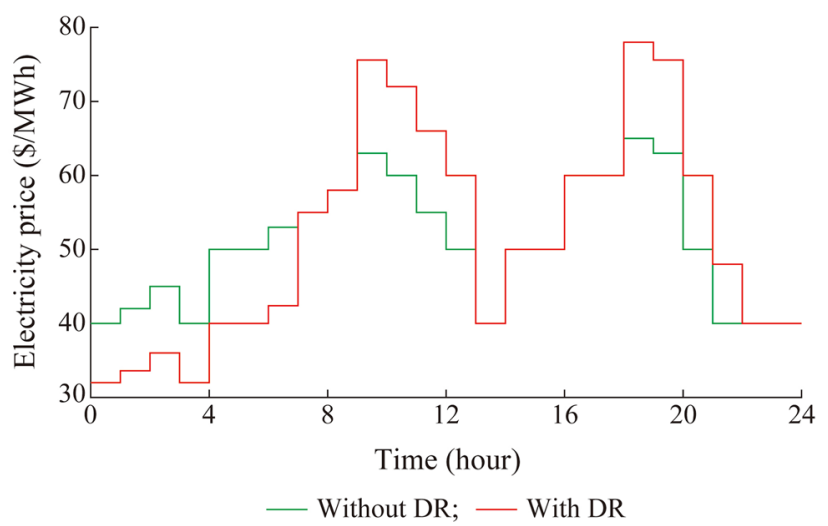

(a) Hourly electricity prices before and after TOU program in normal DR

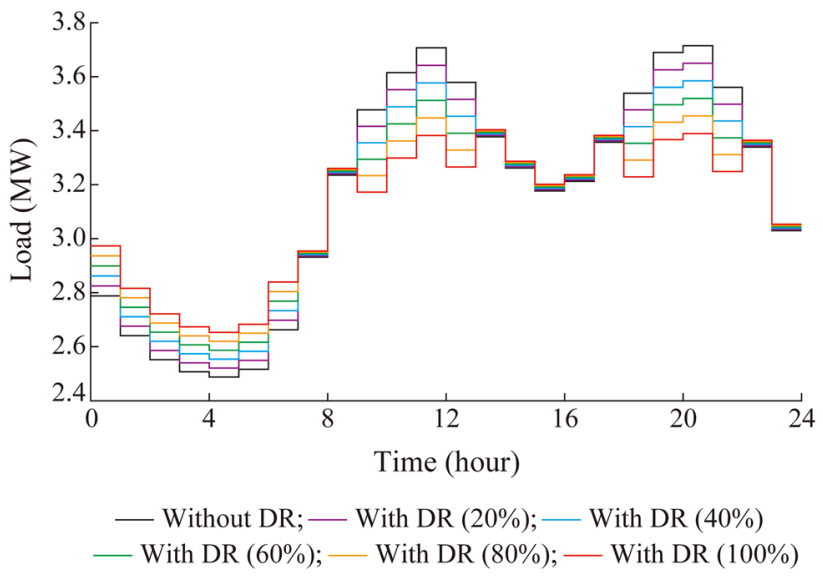

(b) Load profile in presence of TOU DR

Fig. 3 Hourly electricity prices of time of use (TOU) DR and the impact on load profile 


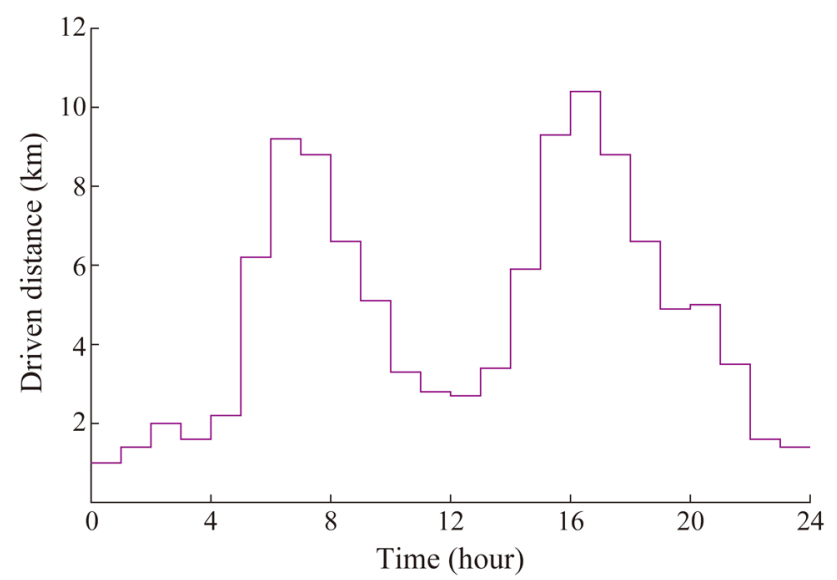

Fig. 4 Daily driven pattern of PEVs' users

Table 2 Installed capacity of units and their availability

\begin{tabular}{lll}
\hline Unit & Installed capacity (kWh) & Availability (p.u.) \\
\hline 1 & 2.70 & 0.9718 \\
2 & 1.30 & 0.8954 \\
3 & 0.70 & 0.8383 \\
4 & 0.20 & 0.9923 \\
5 & 0.30 & 0.9843 \\
6 & 2.25 & 0.9557 \\
7 & 0.25 & 0.9886 \\
8 & 0.20 & 0.9991 \\
\hline
\end{tabular}

Table 3 Self- and cross-elasticity of system load

\begin{tabular}{lrrr}
\hline Period & \multicolumn{1}{l}{ Elasticity } & \\
\cline { 2 - 4 } & \multicolumn{1}{l}{ Valley } & \multicolumn{1}{c}{ Flat } & \multicolumn{1}{c}{ Peak } \\
\hline Valley & -0.030 & 0.010 & 0.012 \\
Flat & 0.010 & -0.050 & 0.016 \\
Peak & 0.012 & 0.016 & -0.050 \\
\hline
\end{tabular}

\section{Case study}

The proposed framework for optimizing the reliability indexes of distribution system utilizing PEVs and RTP DR program is presented in the previous section. For evaluating the effectiveness of the proposed model, firstly IEEE 33-bus radial distribution system [35] is adopted. Figure 2 illustrates the diagram of this system. The hourly electricity price of DR and impact of different participation percentage on load profile are shown in Fig. 3a and b. For greater percentage of customers' participation, peak load is lowered more and load profile is more flattened. PEVs'

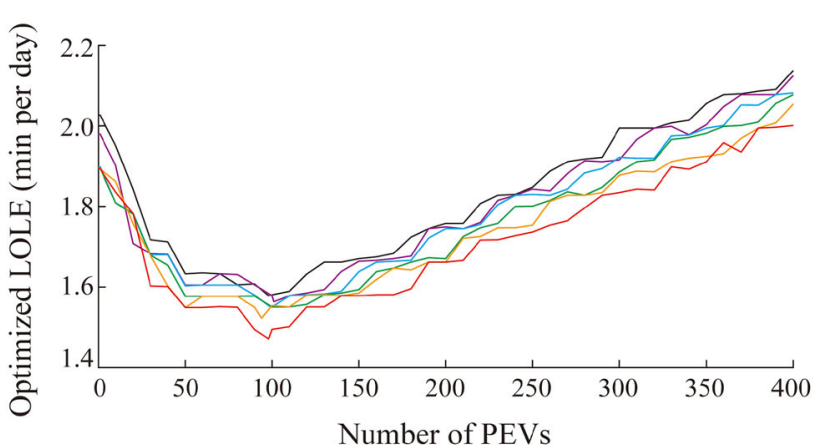

(a) LOLE optimization

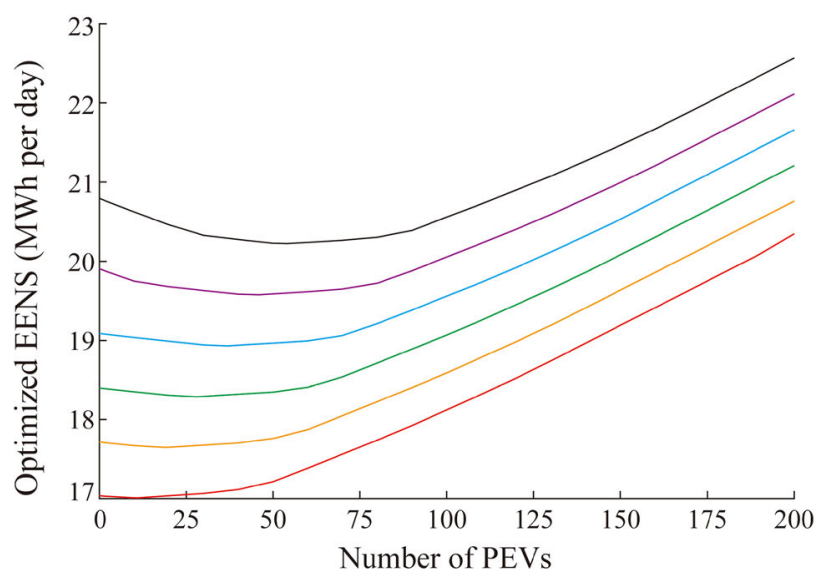

(b) EENS optimization

— Without DR; — With DR $(20 \%) ;-$ With DR $(40 \%)$
With DR $(60 \%) ;$ — With DR $(80 \%) ;-$ With DR $(100 \%)$

Fig. 5 LOLE and EENS optimization with DR and PEVs

consumption is depicted in Fig. 4 [22]. Moreover, units are shown in Table 2. The capacity of each PEV's battery is 25 $\mathrm{kWh}$, and the charger size based on the capacity of current domestic network $(4.6 \mathrm{~kW})$ is considered $4 \mathrm{~kW}$. Self- and cross-elasticity of customers are provided in Table 3.

For the implementation of DR, the time interval between hour 1 and hour 7 is considered as valley hours and the time interval between hour 10 and hour 13. Moreover, hour 19 to hour 22 are selected as peak hours. The other time intervals are considered as flat hours. In this study, it is assumed that $W$ is $6 \mathrm{~km} / \mathrm{kWh}$, i.e. the distance driven by a PEV using $1 \mathrm{kWh}$ energy is $6 \mathrm{~km}$. Accordingly, (24) changes to that $\delta_{h}^{e}$ is equal to $\delta_{h} / 6$, which is used in numerical simulation as vehicle consumption.

\subsection{Reliability}

Results of utilizing PEVs for LOLE and EENS minimization considering TOU-DR and different participation percentage are shown in Fig. 5. For a specified number of PEVs, the reliability indexes are optimized. Both reliability indexes are improved by starting from a low number of 


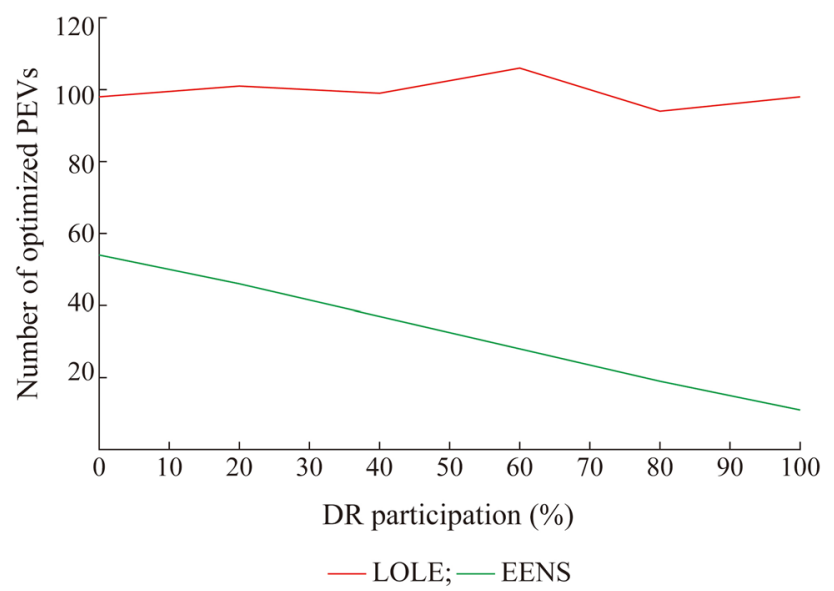

Fig. 6 Optimized number of PEVs in LOLE and EENS optimization

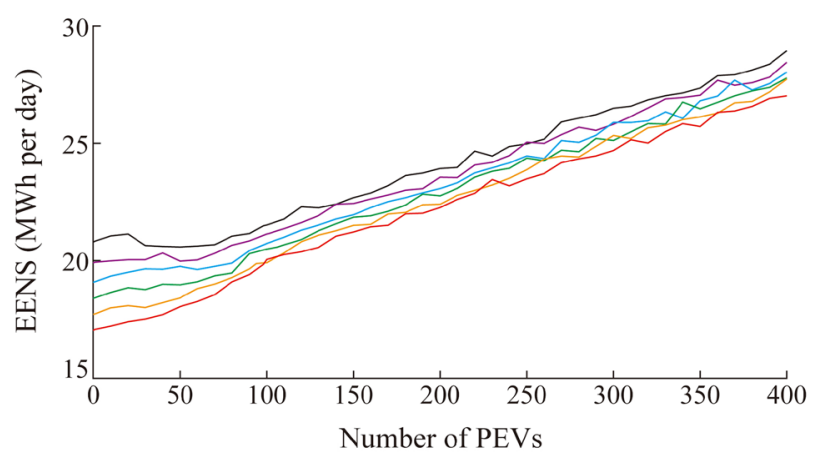

(a) EENS variation based on LOLE optimization

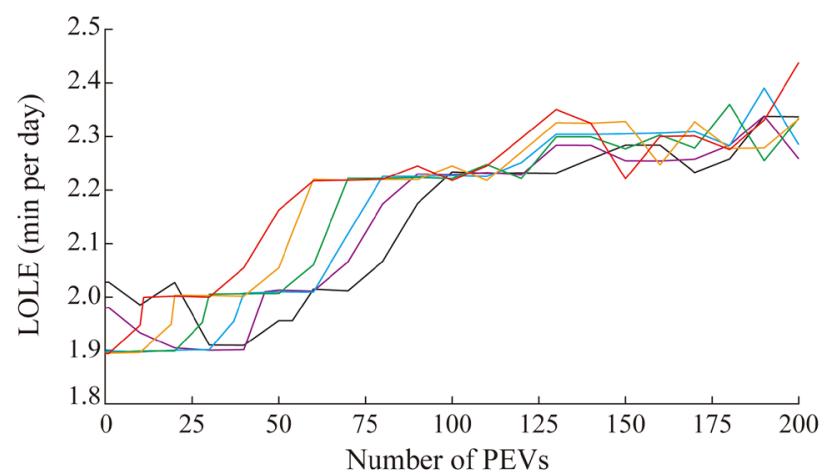

(b) LOLE variation based on EENS optimization

— Without DR; — With DR (20\%); — With DR (40\%)

— With DR (60\%); — With DR $(80 \%)$; — - With DR $(100 \%)$

Fig. 7 LOLE and EENS variation based on optimizing another index

PEVs, and will be deteriorated again for numbers greater than that optimal number. The curves of both indexes are U-shaped such that with increasing $P_{P}$ from 0 to 100 , the indexes are improved. As mentioned, these indexes are optimized adopting PSO algorithm considering related constraints. The optimized number of PEVs for both indexes and for participation of DR are shown in Fig. 6.

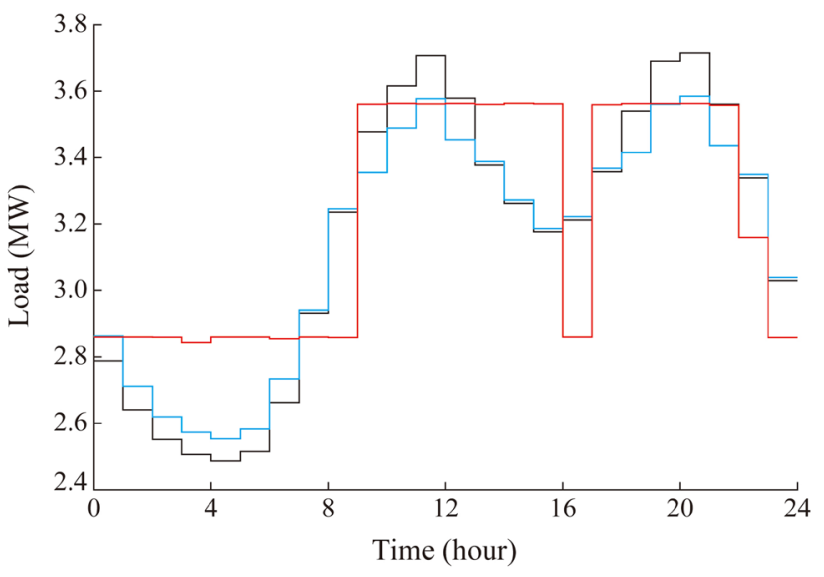

(a) Load profile in LOLE optimization

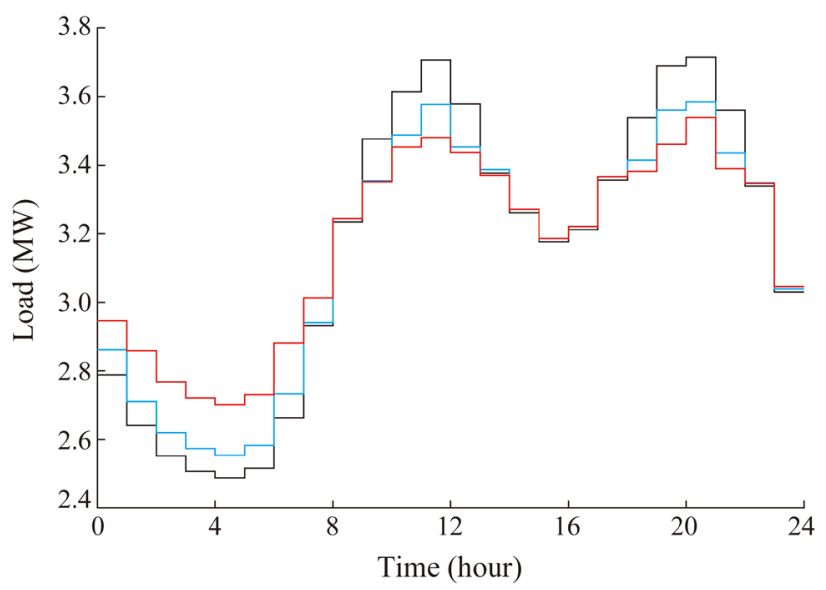

(b) Load profile in EENS optimization

— Without DR and PEVs; — With DR (40\%)

— With DR (40\%) and PEVs

Fig. 8 Load profile in LOLE and EENS optimization with $40 \%$ participation in DR

Variation of EENS based on LOLE optimization is presented in Fig. 7a. EENS is decreased uniformly by increasing LOLE. Also, Fig. 7b illustrates the variation of LOLE based on EENS optimization. Likewise, when optimizing LOLE, LOLE is decreased by increasing EENS. These figures show that such indexes are not sympathy with each other. By optimizing one of LOLE or EENS, the other index would be lower. The requirement of customers determines the index for optimization.

\subsection{Network characteristic}

In this paper, characteristics of the system in presence of optimizing mentioned reliability indexes are presented and analyzed to assess the impact of reliability improvement of radial distribution system using charging/discharging of 


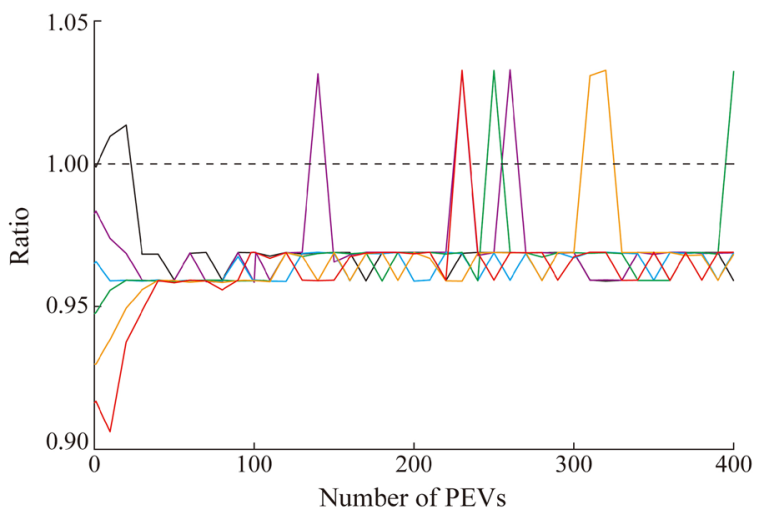

(a) Load peak in LOLE optimization

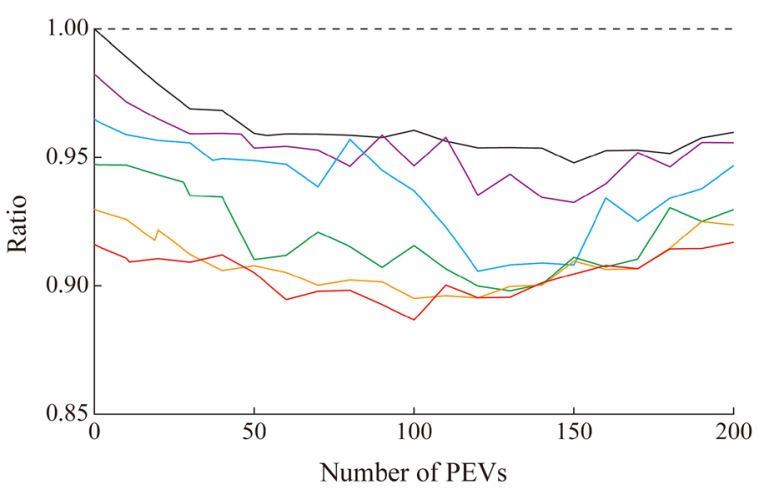

(b) Load peak in EENS optimization

— Without DR; — With DR (20\%); — With DR (40\%)

— With DR (60\%); — With DR (80\%); — With DR (100\%)

Fig. 9 Impact of LOLE and EENS optimization on load peak

PEVs and DR on these characteristics which is important for customers and utilities as other factors of satisfaction. These characteristics contain load profile, load peak, voltage profile and energy loss.

Load profile of system based on LOLE and EENS optimization using PEVs for optimized amount of these two indexes in presence of DR with $40 \%$ participation is shown in Fig. 8. As can be seen, LOLE level the load in two level as far as possible based on the number of PEVs, the capacity, daily consumption and DR program while EENS smooth the load curve by transmit the load from peak hours to valley hours.

The ratio between peak of load with PEVs and DR and peak of the base load in LOLE and EENS optimization is presented in Fig. 9, which shows the better impact of EENS in the viewpoint of load peak comparing to LOLE.

Energy loss of system based on LOLE and EENS optimization in presence of DR is shown in Fig. 10. In LOLE optimization, power loss curve has local

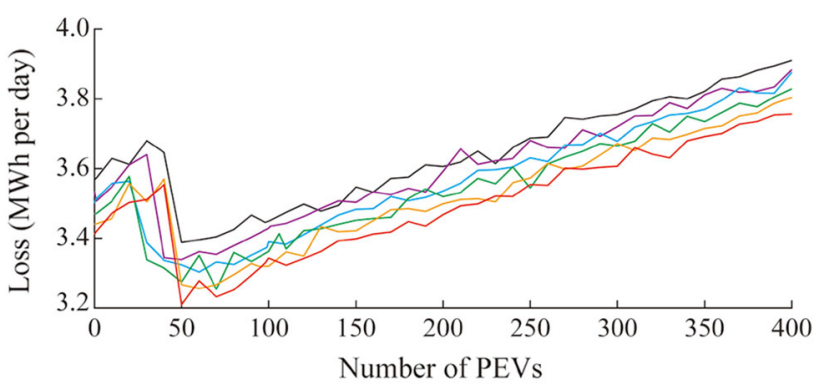

(a) Energy loss in LOLE optimization

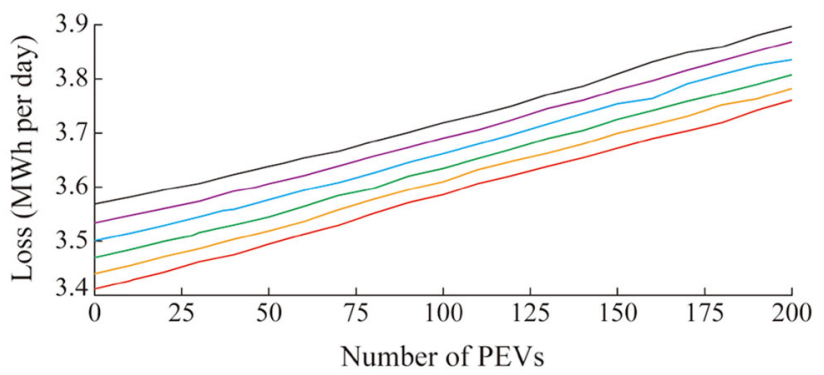

(b) Energy loss in EENS optimization — Without DR; — With DR (20\%); — With DR (40\%)

— With DR $(60 \%)$; — With DR $(80 \%)$; — With DR $(100 \%)$

Fig. 10 Energy loss variation based on LOLE and EENS optimization

minimum and maximum. EENS has no local optimum and increases uniformly by increasing the number of PEVs.

The range of voltage variations of system buses is shown in Fig. 11 which is based on LOLE and EENS optimization using PEVs in presence of DR with $40 \%$ participation for optimized value of these two indexes. The colors are continuous and single-piece which show the voltage profile of all 24 hours such that the black color is at the below of the other two colors, and the green color is at the below of the yellow color.

\section{Reliability improvement prioritizations}

In this section, the prioritization of PEVs and DR for improving the reliability of distribution system is presented. Figure 12a shows the impact of adopting PEVs and implementation of DR on optimized LOLE. For improving LOLE, the impact of adding PEV is much more than the impact of applying DR even for $100 \%$ participation. As it is obvious from these figures, the impact of both PEVs and DR on LOLE of the distribution system, approximately is equal to the impact of only adding PEVs which shows the negligible impact of DR on LOLE. Likewise, Fig. 12b presents the impact of these two factors on EENS that 


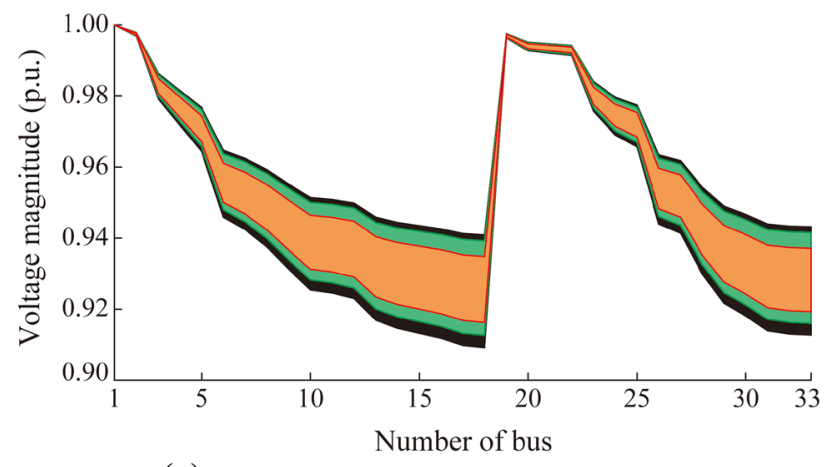

(a) Variation of voltage profile in LOLE optimization

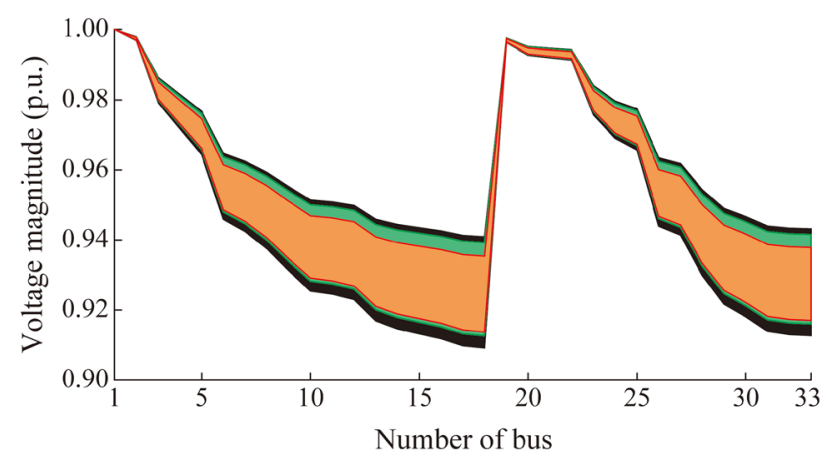

(b) Variation of voltage profile in EENS optimization

Without DR and PEVs; With DR (40\%) With DR (40\%) and PEVs

Fig. 11 Variation of voltage profile with optimized number of PEVs in presence of $40 \%$ participation of DR in LOLE and EENS optimization

shows more effectiveness of DR than PEVs for improving EENS. The impact of both PEVs and DR on distribution system EENS is equal to only implementing DR. However, DR needs low participation percentage for the excess on PEVs, and the impact of both DR and PEVs is equal. Figure 13 shows the impact of DR and PEVs on improvement percentage of LOLE and EENS that prove again the excellence of adding PEVs for improving LOLE and DR for improving EENS.

For improving both reliability indexes, considering both PEVs and DR is a suitable selection, which improves both LOLE and EENS. The prioritization of DR and PEVs for improving reliability indexes is presented in Tables 4 and 5. As in Table 4, for improving LOLE, there are three choices of prioritizing. Table 5 shows that for improving EENS, there are four choices. As shown in the table, from $0 \%$ to $12.84 \%$ DR participation, the impact of PEVs is greater, while for $12.83 \%$, the impact of both PEVs and DR is equal. Moreover, for participation percentage greater than $12.83 \%$, the impact of DR is greater than PEVs. Accordingly, DR has a great impact on improving EENS while PEVs has no considerable impact on such reliability

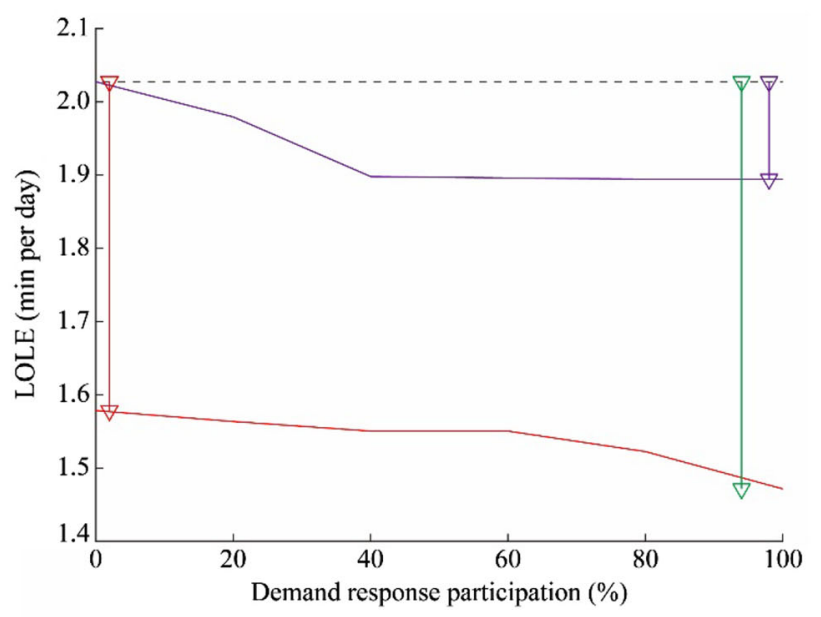

(a) Impact of PEVs and DR on LOLE

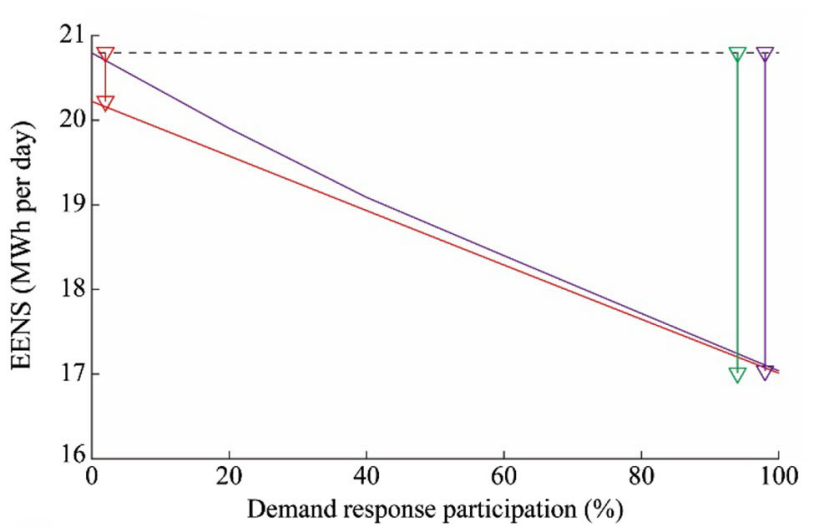

(b) Impact of PEVs and DR on EENS

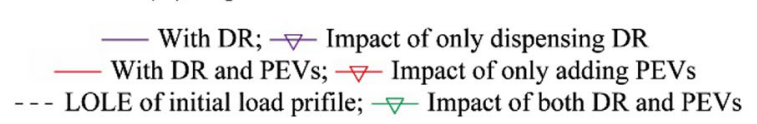

Fig. 12 Impact of PEVs and DR on LOLE and EENS

index. Also, Fig. 13 shows the impact of ODR on indexes. As shown in this figure, in the case of adopting ODR instead of normal DR, LOLE is improved and has an impact on EENS, i.e. the result of EENS optimization in ODR is exactly the same solution of DR while LOLE is decreased with no considerable percentage in improvement.

\section{Conclusion}

In this paper, reliability indexes optimization of radial distribution system is accomplished utilizing PEVs and DR as well as prioritizing these two factors. In addition, a stochastic framework is proposed for connecting PEVs to buses and a new formulation to calculate the COPT. The effectiveness of the proposed scheme is proved by adopting 


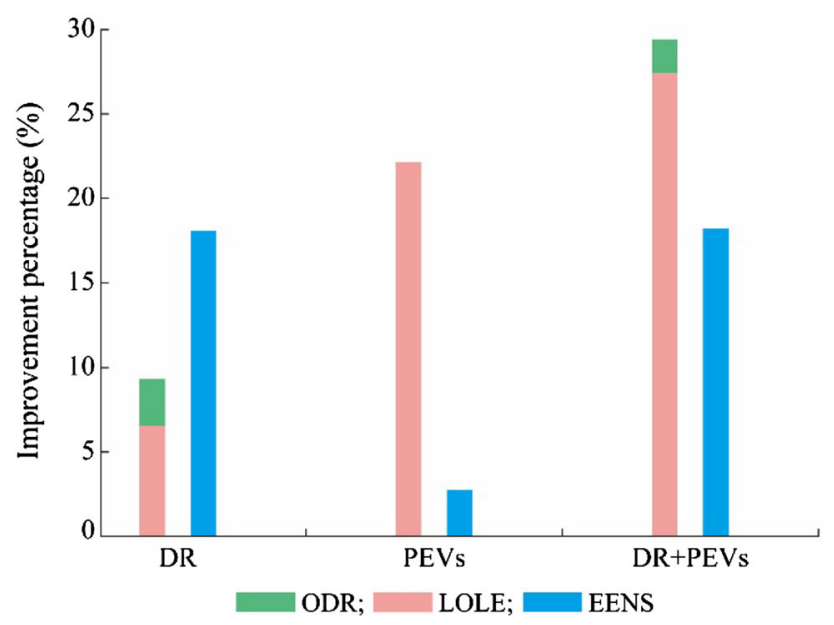

Fig. 13 Improvement percentage of LOLE and EENS by PEVs and DR

Table 4 Prioritization of PEVs and DR for improving LOLE

\begin{tabular}{lll}
\hline Priority & Factors & Improvement (\%) \\
\hline First & Implementing DR and adding PEVs & $22.15-27.42$ \\
Second & Adding PEVs & 22.15 \\
Third & Implementing DR & $0-6.57$ \\
\hline
\end{tabular}

Table 5 Prioritization of PEVs and DR for improving EENS

\begin{tabular}{|c|c|c|}
\hline Priority & Factors & $\begin{array}{l}\text { Improvement } \\
(\%)\end{array}$ \\
\hline First & Implementing DR and adding PEVs & $18.07-18.20$ \\
\hline Second & Implementing DR (12.84\%-100\%) & $2.75-18.07$ \\
\hline Third & $\begin{array}{l}\text { Implementing DR (12.84\%) or adding } \\
\text { PEVs }\end{array}$ & 2.075 \\
\hline Forth & Implementing DR (0-12.84\%) & 2.075 \\
\hline
\end{tabular}

the IEEE 33-bus radial distribution system. The results are analyzed in viewpoint of number of vehicles, load curve, voltage profile and loss. Due to the superiority of PSO to mathematical methods, this algorithm is employed to optimize the reliability indexes.

Open Access This article is distributed under the terms of the Creative Commons Attribution 4.0 International License (http:// creativecommons.org/licenses/by/4.0/), which permits unrestricted use, distribution, and reproduction in any medium, provided you give appropriate credit to the original author(s) and the source, provide a link to the Creative Commons license, and indicate if changes were made.

\section{References}

[1] Sadeghian O, Oshnoei A, Nikkhah S et al (2019) Multi-objective optimization of generation maintenance scheduling in restructured power systems based on global criterion method. IET Smart Grid. https://doi.org/10.1049/iet-stg.2018.0140

[2] Federal energy regulatory commission (2006) Regulatory commission survey on demand response and time based rate programs/tariffs. http://www.FERC.gov

[3] Kirschen DS, Strbac G, Cumperayot P et al (2000) Factoring the elasticity of demand in electricity prices. IEEE Trans Power Syst 15(2):612-617

[4] Nazari-Heris M, Abapour S, Mohammadi-Ivatloo B (2017) Optimal economic dispatch of FC-CHP based heat and power micro-grids. App Therm Eng 114:756-769

[5] Rahmani-Andebili M (2016) Modeling nonlinear incentivebased and price-based demand response programs and implementing on real power markets. Electr Power Syst Res 132:115-124

[6] Haghi V, Qu Z (2018) A Kernel-based predictive model of EV capacity for distributed voltage control and demand response. IEEE Trans Smart Grid 9(4):3180-3190

[7] Yu R, Zhong W, Xie S et al (2016) Balancing power demand through EV mobility in vehicle-to-grid mobile energy networks. IEEE Trans Ind Inform 12(1):79-90

[8] McKenna K, Keane A (2016) Residential load modeling of price-based demand response for network impact studies. IEEE Trans Smart Grid 7(5):2285-2294

[9] Gutiérrez-Alcaraz G, Tovar-Hernández JH, Lu CN (2016) Effects of demand response programs on distribution system operation. Int J Electr Power Energy Syst 74:230-237

[10] Wang F, Xu H, Xu T et al (2017) The values of market-based demand response on improving power system reliability under extreme circumstances. Appl Energy 193:220-231

[11] Aghaei J, Alizadeh MI, Siano P et al (2016) Contribution of emergency demand response programs in power system reliability. Energy 103:688-696

[12] Zeng B, Wu G, Wang J et al (2017) Impact of behavior-driven demand response on supply adequacy in smart distribution systems. Appl Energy 202:125-137

[13] García-Villalobos J, Zamora I, San Martín JI et al (2014) Plugin electric vehicles in electric distribution networks: a review of smart charging approaches. Renew Sustain Energy Rev 38:717-731

[14] Haidar AMA, Muttaqi KM, Sutanto D (2014) Technical challenges for electric power industries due to grid-integrated electric vehicles in low voltage distributions: a review. Energy Convers Manag 86:689-700

[15] Habib S, Kamran M, Rashid U (2015) Impact analysis of vehicle-to-grid technology and charging strategies of electric vehicles on distribution networks-a review. J Power Sources 277:205-214

[16] Yang Z, Li K, Foley A (2015) Computational scheduling methods for integrating plug-in electric vehicles with power systems: a review. Renew Sustain Energy Rev 51:396-416

[17] Rahman I, Vasant PM, Singh BSM et al (2016) Review of recent trends in optimization techniques for plug-in hybrid, and electric vehicle charging infrastructures. Renew Sustain Energy Rev 58:1039-1047

[18] Rajabi M, Esmaili M (2014) Optimal charging of plug-in electric vehicles observing power grid constraints. IET Gener Transm Distrib 8(4):583-590

[19] Jian L, Zheng Y, Xiao X et al (2015) Optimal scheduling for vehicle-to-grid operation with stochastic connection of plug-in electric vehicles to smart grid. Appl Energy 146:150-161 
[20] Oshnoei A, Hagh MT, Khezri R et al (2017) Application of IPSO and fuzzy logic methods in electrical vehicles for efficient frequency control of multi-area power systems. In: Proceedings of 2017 Iranian conference on electrical engineering (ICEE), Tehran, Iran, 2-4 May 2017, 6pp

[21] Khezri R, Oshnoei A, Hagh Tarafdar et al (2018) Coordination of heat pumps, electric vehicles and AGC for efficient LFC in a smart hybrid power system via SCA-based optimized FOPID controllers. Energies 11(2):420

[22] Božič D, Pantoš M (2015) Impact of electric-drive vehicles on power system reliability. Energy 83:511-520

[23] Xu NZ, Chung CY, Member S (2015) Reliability evaluation of distribution systems including vehicle-to-home and vehicle-togrid. IEEE Trans Power Syst 31(1):759-768

[24] Reddy GH, Goswami AK, Dev Choudhury NB (2018) Impact of plug-in electric vehicles and distributed generation on reliability of distribution systems. Eng Sci Technol Int J 21(1):50-59

[25] Schweppe FC, Caramanis MC, Tabors RD et al (1989) Spot pricing of electricity. Kluwer Academic Publishers, Dordrecht

[26] Kirschen DS, Strbac G (2004) Fundamentals of power system economics. Wiley, Hoboken

[27] Staff report to minister of energy (2004) Demand-side management and demand response in the Ontario electricity sector. http://www.ontla.on.ca/library/repository/mon/8000/243395.pdf

[28] Goel L, Wu Q, Wang P (2007) Reliability enhancement and nodal price volatility reduction of restructured power systems with Stochastic demand side load shift. In: Proceedings of 2007 IEEE PES general meeting, Tampa, USA, 24-28 June 2007, 8pp

[29] Aalami HA, Moghaddam MP, Yousefi GR (2010) Modeling and prioritizing demand response programs in power markets. Electr Power Syst Res 80(4):426-435

[30] Aalami HA, Parsa Moghaddam M, Yousefi GR (2015) Evaluation of nonlinear models for time-based rates demand response programs. Int J Electr Power Energy Syst 65:282-290

[31] Billinton R (1996) Reliability evaluation of power systems, 2nd edn. Plenum Press, New York

[32] Shirmohammadi D, Hong HW, Semlyen A et al (1988) A compensation-based power flow method for weakly meshed distribution and transmission networks. IEEE Trans Power Syst 3(2):753-762

[33] Kennedy J, Eberhart R (1995) Particle swarm optimization. In: Proceedings of IEEE international conference on neural networks, Perth, Australia, 27 November-1 December 1995, 7pp

[34] Clerc M, Kennedy J (2002) The particle swarm-explosion, stability, and convergence in a multidimensional complex space. IEEE Trans Evol Comput 6(1):58-73
[35] Oshnoei A, Khezri R, Tarafdar HM et al(2018) Direct probabilistic load flow in radial distribution systems including wind farms: an approach based on data clustering. Energies 11(2):310

Omid SADEGHIAN received the B.Sc. and M.Sc. degrees in power engineering from the University of Kordestan and University of Tabriz, Iran, respectively. His research interests include virtual power plants, probabilistic load flow, planning, demand response, energy storage placement, and generation maintenance scheduling.

Morteza NAZARI-HERIS received the B.Sc. and M.Sc. degrees in power engineering from the University of Tabriz, Tabriz, Iran, in 2015 and 2017. He is currently working as research assistant at the same university. His research interests include integrated energy systems, economics, planning, and management of electrical energy systems, micro grids, energy analysis and efficiency, and fault current limiters.

Mehdi ABAPOUR received his M.Sc. and Ph.D. degrees in electrical engineering from University of Tabriz and Tarbiat Modares University, in 2007 and 2013, respectively. He is currently an Associate Professor with the Faculty of Electrical and Computer Engineering, University of Tabriz, Tabriz, Iran. His research interests include stochastic power system analysis, reliability of power electronic systems and power systems, power system dynamics, FACTS devices and fault current limiters.

S. Saeid TAHERI received his B.Sc. and M.Sc. degrees in electrical engineering from Shahid Beheshti University and Tehran Polytechnic, Iran, respectively, and Ph.D. Degree from University of Mohaghegh Ardabili in 2017. Currently, he is an Assistant Professor at Islamic Azad University, Sarab Branch, Iran. His research interests mainly include the study of mathematical or heuristic modellings which is related to electrical problems within different environments using multi-level optimization models, during these years.

Kazem ZARE received the B.Sc. and M.Sc. degrees in electrical engineering from University of Tabriz, Tabriz, Iran, in 2000 and 2003, respectively, and Ph.D. degree from Tarbiat Modares University, Tehran, Iran, in 2009. Currently, he is an Associate Professor of the Faculty of Electrical and Computer Engineering, University of Tabriz, Tabriz, Iran. His research areas include power system economics, distribution networks, microgrid and energy management. 